\title{
Marsh, McLuhan and the Crystallographic Message
}

Larry R. Falvello

University of Zaragoza - CSIC, Department of Inorganic Chemistry and Aragón

Materials Science Institute, Pedro Cerbuna 12, 50009 Zaragoza, Spain

falvello@unizar.es

ABSTRACT. A quote from McLuhan is used as the point of departure for a discussion of the extent to which our understanding of the results of small-molecule structure analysis is influenced by the way the analyses are done and the way the results are communicated. New means of presenting crystallographic results, exemplified by tools such as the increasingly popular Hirshfeld surfaces, invite researchers to revisit results that were obtained before these methods became available. With such tools in combination with the increasing on-line archival of data and results, it can be foreseen that post-publication analysis by the original authors or by others will become a common practice in crystallography. Applying Marsh's example of posterior re-evaluation of results, and considering specific structural chemistry problems and recently available crystallographic tools, it is concluded that the practice of making experimental data available with a publication provides the potential for future added value to the data. 